\begin{tabular}{|c|c|c|}
\hline Beitr. Ent. & Keltern & ISSN 0005-805X \\
\hline $\mathbf{5 6}(2006) 2$ & S. $465-475$ & 15.12 .2006 \\
\hline
\end{tabular}

\title{
Die Verbreitung der mitteleuropäischen Arten der Simulium variegatum Gruppe
}

\author{
(Diptera: Simuliidae)
}

Mit 8 Figuren

Matúš Kúdela

Summary

This paper describes the occurrence of Simulium argyreatum MeIgen, 1838, Simulium maximum (KNoz, 1961), Simulium monticola Friederichs, 1920, Simulium variegatum Meigen, 1818 and the not described taxon S. sp. aff. monticola in general, and more detailed in the territory of Slovakia. S. variegatum, S. argyreatum und S. monticola have large areas differing in some details (e.g. occurrence in the British Islands, Skandinavien, Northafrica). S. maximum is restricted to the higher mountains in Central and Southern Europe; $S$. sp. aff. monticola is known only from Slovakia till now. S. variegatum and S. argyreatum are common in Slovakia; the formerly published wide occurrence of $S$. monticola has not been confirmed. Revised data shows, that $S$. maximum is more common, than suspected. $S$. argyreatum is widely distributed through all altitudes. S. variegatum prefers lower (200-800 m a.s.l.) and S. maximum higher altitudes $(600-1000 \mathrm{~m}$ a.s.l.) than $S$. monticola and $S$. sp. aff. monticola (400-800 m a.s.l.).

\section{Zusammenfassung}

Die Gesamtverbreitung und eine detaillierte Verbreitung in der Slowakei von den Arten Simulium argyreatum Meigen, 1838, Simulium maximum (KNoz, 1961), Simulium monticola Friederichs, 1920, Simulium variegatum Meigen, 1818 und dem noch nicht beschriebenen Taxon S. sp. aff. monticola wird angegeben. S. variegatum, S. argyreatum und S. monticola haben umfangreiche Areale, die sich in einigen Details (z. B. Vorkommen auf den Britischen Inseln, in Skandinavien, Nordafrika) unterscheiden. S. maximum ist auf höhere Gebirge Mittel- und Südeuropas beschränkt. S. sp. aff. monticola ist bis jetzt nur aus der Slowakei bekannt. S. variegatum und S. argyreatum sind in der Slowakei weit verbreitet. Die früher angenommene weite Verbreitung von S. monticola wurde nicht bestätigt. Die nachgeprüften Angaben zeigen, daß S. maximum häufiger ist, als bis jetzt publiziert wurde. S. argyreatum ist über alle Höhenzonen weit verbreitet. S. variegatum bevorzugt niedrigere Lagen (200-800 m ü. M.) und S. maximum höhere Lagen (600-1000 m ü. M.) als S. monticola und $S$. sp. aff. monticola (400-800 m ü. M.).

\section{Keywords}

Simulium variegatum group, occurrence, Central Europe 


\section{Einleitung}

Weltweit sind 36 Arten aus der Simulium variegatum Gruppe (Diptera: Simuliidae) bekannt. Die meisten Arten sind paläarktisch, nur einige Arten kommen auch in der orientalischen Region vor. In Mitteleuropa wurden vier Arten beschrieben: Simulium argyreatum MeIgen, 1838, Simulium maximum (KNOz, 1961), Simulium monticola Friederichs, 1920 und Simulium variegatum MeIgen, 1818. Bis auf S. maximum sind sie weit verbreitet. S. maximum ist nur aus einigen Gebirgen Mittel- und Südeuropas bekannt. Drei weitere europäische Arten besiedeln einen spezifisch begrenztes Areal: $S$. xanthinum Edwards, 1933 Südwesteuropa und Marokko (CrosskeY, 1991), S. sicanum (Rivosecchi, 1962) Sizilien und Süditalien (Rivosecchi, 1978) und S. laplandicum (Tschubareva \& Jankovskij, 1992) den Norden des europäischen Teiles Russlands (Jankovskij, 2002, Tschubareva \& JankovskiJ, 1992). Weitere Arten können von Osten bis zum Kaukasus-Gebiet vordringen. Die Funde (bzw. das Vorkommen) von S. debacli Terteryan, 1952, S. exile (Rubtsov, 1956) und S. monticoloides (Rubtsov, 1956) wurden aus Rumänien und Bulgarien publiziert (Crosskey \& Howard, 2004, Dinulescu, 1966). Die Identität dieser Funde wäre zu überprüfen.

Die Arten der S. variegatum Gruppe sind in den Kriebelmückengemeinschaften Mitteleuropas dominant (z. B. IlléŠovÁ \& Halgoš, 1999, 2004, IlléšovÁ \& JedLIČKA, 1992, Illéšová u. a., 2000). Bei der Bewertung der Kriebelmückenbiodiversität der Slowakei gehören sie zu den Arten mit der größten Anzahl der Fundorte und der bewohnten Gebietseinheiten (JedličKa u. a., 2001).

Allerdings ist ein Teil der Angaben wahrscheinlich dubios - vor allem wegen der Probleme mit der Bestimmung und Identität einzelner Arten. Zu den größten Schwierigkeiten gehören die vertauschte Identität von $S$. monticola und $S$. argyreatum (JeDličKA u. a., 2004), die geringen Unterschiede zwischen S. monticola und S. maximum (Crosskey \& Crosskey, 2000), neuere Befunde, daß die Unterscheidungsmerkmale nicht eindeutig verwendet werden können - z. B. bei den Larven von $S$. variegatum und $S$. argyreatum (BAss, 1998) und die Existenz von einem unbeschriebenen Taxon in Mitteleuropa (S. sp. aff. monticola, siehe auch weiter, Dušinskŕ u. a., 2006).

In dieser Arbeit versuchen wir eigene und publizierte Angaben über die Verbreitung der mitteleuropäischen Arten der S. variegatum Gruppe zusammenzufassen. Bei der Analyse berücksichtigen wir die neuen Kenntnisse über die vorkommende Taxa (z. B. DušınskÝ u. a., 2006, JedLIČKa u. a., 2004, KúdeLA \& JeDLIČKA, 2002), und insbesondere befassen wir uns mit der Verbreitung in der Slowakei und ihrer Höhenzonenverbreitung.

\section{Material und Methoden}

Wir haben publizierte Angaben, sowie das Material aus den Sammlungen der Comenius Universität und eigenes Material verwendet. Beim Bearbeiten des Sammlungsmaterials haben wir fünf Taxa unterschieden: S. variegatum, S. argyreatum, S. maximum, S. monticola und $S$. sp. aff. monticola. Die als $S$. sp. aff. monticola bezeichnete Art (Dušınskŕ u. a., 2006) entspricht der früher angegebenen morphologischen Form S. monticola 1 (z. B. Kúdela \& JedličKa, 2002).

Die Gesamtverbreitung der Arten haben wir aus publizierten Unterlagen zusammengefaßt, z. T. handelt es sich um sekundäre Quellen. Die Gesamtverbreitung wurde nur 
grob - auf der Basis einzelner Länder kartiert. Eine detaillierte Verbreitung wird nach Klärung des taxonomischen Status bzw. der Identität einiger Populationen sinnvoll. Die publizierten Angaben haben wir, wo es möglich war, nachgeprüft (Deutschland) und mit neuen Angaben ergänzt (Griechenland).

Bei der detaillierten Kartierung des Vorkommens in der Slowakei gehen wir bei allen Arten von eigenen (insgesammt etwa 77 Kriebelmückenfundorte) oder nachgeprüften Sammlungsangaben aus (insgesammt etwa 312 Kriebelmückenfundorte). Bei S. argyreatum und $S$. variegatum haben wir auch publizierte Unterlagen verwendet (insgesamt etwa 636 Kriebelmückenfundorte). Alle Angaben über die Verbreitung von S. monticola, $S$. maximum und $S$. sp. aff. monticola basieren auf der Bestimmung von Puppen, da diese problemlos zu unterscheiden sind. Die Verbreitung in der Slowakei ist auch auf der Basis der 92 geomorphologischen Einheiten (Gliederung der Karpaten) angegeben (MAzúr \& LuKNiš, 1978).

Die hypsometrische Analyse des Vorkommens wurde an den Verbreitungsdaten aus der Slowakei gemacht. Angaben, die ohne Meereshöhe des Fundortes publiziert wurden und die auch sekundär nicht festgelegt wurden, wurden nicht verwendet. Die Abhängigkeit des Vorkommens von der Meereshöhe wurde als eine quadratische Regression $Y=\beta_{0}+\beta_{1} X+\beta_{2} X^{2}$ festgestellt. Die Wahrscheinlichkeit des Vorkommens in einer Meereshöhe ist als eine logistische Funktion $\mathrm{p}=\left(\mathrm{e}^{\beta 0+\beta 1 \mathrm{X}+\beta 2 \mathrm{X}^{\prime}}\right) /\left(1+\mathrm{e}^{\beta 0+\beta 1 \mathrm{X}+\beta 2 \mathrm{X}^{\prime}}\right)$ angegeben.

Insgesamt wurde die hypsometrische Verbreitung von $S$. variegatum und $S$. argyreatum an 491 Kriebelmückenfundorten analysiert, die Angaben über das Vorkommen kommen aus publizierten Quellen (BASTl u. a., 1992, Bulánková \& Halgoš, 1995, Bulánková u. a., 2000, 2001, 2002, Halgoš \& JedličKa, 1974, Harvančí́ \& Topercer, 1985, IlléŠOVÁ, 1989, 1995, IlléŠOVÁ \& Halgoš, 1999, 2002, IlléŠOVÁ \& JEDLIČKA, 1992, IlléŠOvá u. a., 1994, 1995, 2000, JeDliČKA 1970a, 1976, 1982c, 1984, JEDLIČKA \& Trpiš 1971, Krno 1978, 1982, 1995, Krno u. a., 1993, 1994a, 1994b, KubovČík \& Novikmec, 2001, Nováк, 1956, Овв, 1956). Die revidierten S. monticola, S. maximum und $S$. sp. aff. monticola wurden an 266 Kriebelmückenfundorten analysiert (ein großer Teil entspricht den vorigen Fundorten).

\section{Ergebnisse und Diskussion}

\section{Die Gesamtverbreitung}

Die Art S. variegatum hat Meigen 1818 von Material aus Deutschland beschrieben (Crosskey \& Howard, 2004). Ihr Verbreitungsgebiet umfaßt beinahe ganz Europa (nicht Skandinavien), Nordafrika und den Vorderen Osten (Crosskey \& Howard, 2004, GlatthaAr, 1978, Knoz \& JedličKa, 1997, Rivosecchi, 1978, Zwick, 1974, 1993).

Auch $S$. argyreatum wurde anhand von Material aus Deutschland beschrieben (Crosskey \& Howard, 2004). Anschließend wurde die Art in der Mehrheit der europäischen Länder festgestellt: Andorra, Bosnien, Bulgarien, Finnland, Frankreich, Großbritannien, Irland, Italien, Mazedonien, Luxemburg, Norwegen, Österreich, Polen, Portugal, Tschechien, Rumänien, Schweiz, Serbien, Slowakei, Slowenien, Spanien und Ungarn (Crosskey \& Howard, 2004, GlatthaAR, 1978, Knoz \& JedličKa, 1997, Rivosecchi, 1978, Zwick, 1974, 1993). Das Vorkommen der Art wurde wahrscheinlich auch aus Russland publiziert - in den Arbeiten von Rubzow $(1956,1964)$ befinden sich unter dem Namen 
S. monticola nur Abbildungen von S. argyreatum. Die Angaben aus Ungarn (Crosskey \& HowArd, 2004, PAPp, 2001) sind unsicher - in der Arbeit von Rubzow (1967) wurde unter dem Namen $S$. argyreatum das Vorkommen von S. noelleri FrIEDERICHS, 1920 publiziert. Ebenfalls unsicher ist auch die Identität eines als Simulia argyreata $\mathrm{Mg}$. angegebenen Fundes aus Ungarn (Thalhammer, 1900), die Lage der Lokalität (Pomáz) spricht eher dagegen.

S. monticola wurde aus Deutschland beschrieben, die Typenlokalität befindet sich im Harz, Umgebung von Braunlage (KNOz, 1961, Zwick, 1974). Anschließend wurde die Art in der Mehrheit der europäischer Länder (und in Nordafrika) bestätigt: Algerien, Andorra, Belgien, Bosnien, Bulgarien, Tschechien, Frankreich, Italien, Mazedonien, Luxemburg, Noren, Polen, Portugal, Österreich, Rumänien, Serbien, Slowakei, Slowenien, Spanien, Schweiz, Schweden (Crosskey \& Howard, 2004, GlatthaAr, 1978, Knoz \& JedličKa, 1997, MinÁŘ \& Christova, 1971, RivosecCHi, 1978, Zwick, 1974, 1993). Sie fehlt auf den Britischen Inseln. Bei dem Vorkommen in Russland (Rubzow 1956, 1964) handelt es sich wahrscheinlich um S. argyreatum. Wir haben die Art auch in Griechenland festgestellt. Die neuen Funde von S. monticola: 5. Oktober 1995, Monte Renoso, Griechenland - 1 Puppe (leg. Halgoš), 7. Oktober 1995, Porto, Griechenland - 1 Puppe (leg. Halgoš).

S. maximum wurde aus Tschechien beschrieben, die Typenlokalität befindet sich in Jeseníky, ein Teil der Typenserie stammt auch aus den Beskyden (KNOz, 1961). Ihre bekannte Verbreitung beschränkt sich auf auf Mittel- und Südeuropa: Bosnien, Bulgarien, Tschechien, Deutschland, Frankreich, Österreich, Italien, Polen, Rumänien, Schweiz, Serbien, Slowakei und Spanien (Bernard, 1970, Crosskey \& Howard, 2004, Doby, 1963, Glatthaar, 1978, Knoz \& Jedlička, 1997, Rivosecchi, 1978, Seitz, 1992, Zwick, 1974, 1993). Das wirkliche Areal umfaßt oft nur einen kleinen Teil des Landes - z. B. in Deutschland ist $S$. maximum nur aus einigen Gebirgen im Süden bekannt (Seitz, 1992, Zwick, 1974).

Das Vorkommen des noch unbeschriebenen Taxon $S$. sp. aff. monticola ist nur aus verschiedenen Gebirgen in den Westkarpaten (Slowakei) bekannt. In der Literatur wurden keine Beschreibungen oder Abbildungen gefunden, die auf das Vorkommen dieses Taxon hinweisen.

Das Wissen über die Areale dieser Arten ist insofern problematisch - einige Gebiete wurden bisher nicht ausreichend faunistisch untersucht (Balkan, Nordafrika, Westasien). Aufgrund der komplizierten taxonomischen Situation dürften die Arten in einigen Arbeiten nicht verläßlich identifiziert worden sein.

\section{Verbreitung in der Slowakei}

S. variegatum wurde in der Slowakei in 36 geomorphologischen Einheiten gefunden (Fig. 1). Sie fehlte vor allem in den Einheiten mit wenigen Fundorten von Kriebelmücken, wahrscheinlich unabhängig vom Gebiet. Die Art gehört zu den häufigsten in der Slowakei (Frequenz an Lokalitäten 0,12, in den Einheiten 0,48 - JeDLIČKA u. a., 2001).

$S$. argyreatum ist in der Slowakei weit verbreitet (Fig. 2). Fundorte befinden sich in 34 geomorphologischen Einheiten und schließen die höchsten Gebirge (Tatra), sowie einige Niederungen (Borská nížina, Podunajská rovina) ein. Sie wurde vor allem in den Einheiten mit wenigen Lokalitäten von Kriebelmücken nicht gefunden. Mit der Frequenz 0,18 an den Lokalitäten ist $S$. argyreatum die zweithäufigste Kriebelmücke in der Slowakei (S. ornatum Meigen, 1818 hat die Frequenz 0,41; JedličKa u. a., 2001). 


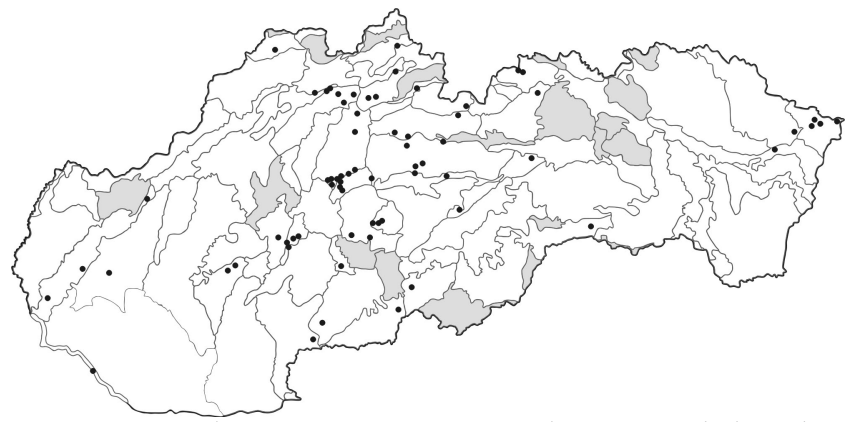

Fig. 1: Die Fundorte von S. variegatum in den geomorphologischen Einheiten der Slowakei. Publizierte und eigene Angaben. Hellgrau: Einheiten ohne bekannte Kriebelmückenfundorte.

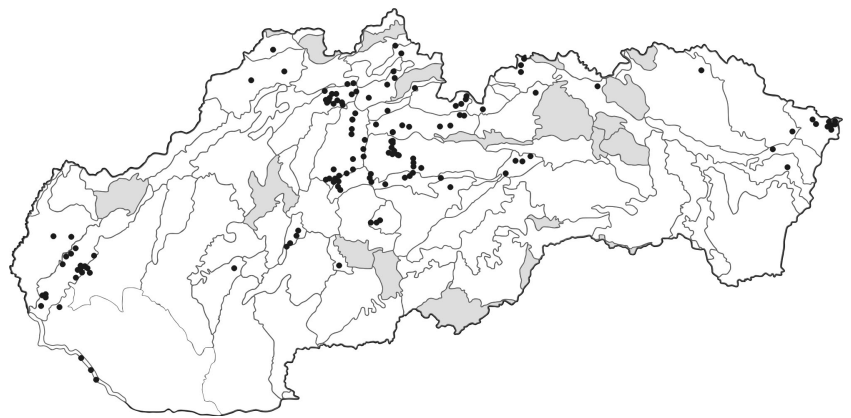

Fig. 2: Die Fundorte von S. argyreatum in den geomorphologischen Einheiten der Slowakei. Publizierte und eigene Angaben. Hellgrau: Einheiten ohne bekannte Kriebelmückenfundorte.

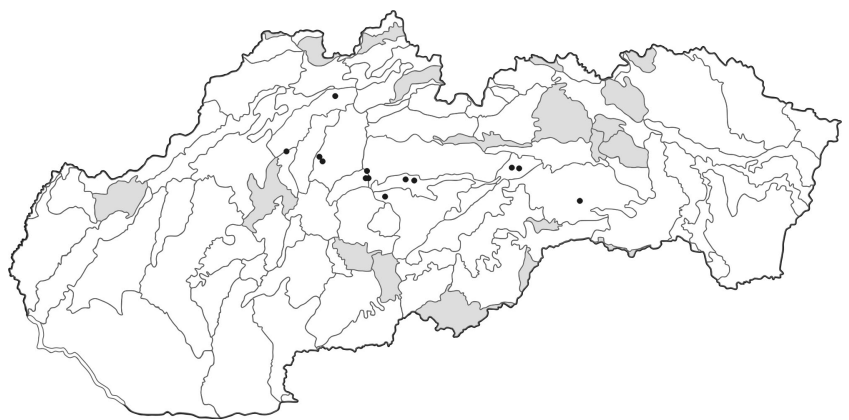

Fig. 3: Die Fundorte von S. monticola in den geomorphologischen Einheiten der Slowakei. Publizierte und eigene Angaben. Hellgrau:

Einheiten ohne bekannte Kriebelmückenfundorte. nur mit dem Schwerpunkt
des Vorkommens in höheren Lagen. Mit den Frequenzen des Vorkommens an den Lokalitäten $(0,17)$ und in den geomorphologischen Einheiten $(0,45)$ gehört sie zu den weit verbreiteten Arten ein (JedLIČKa u. a., 2001). Das Vorkommen von S. maximum wurde aus 11 geomorphologischen Einheiten publiziert, es handelt sich um die höchsten karpathischen Gebirge. Die Frequenzen an den Lokalitäten $(0,06)$ und in den geomorphologischen Einheiten $(0,14)$ entsprechen weder den häufigen noch den seltenen Arten (JeDličKa u. a., 2001). Im nachgeprüften Material dieser drei Arten (S. monticola, S. maximum, $S$. sp. aff. monticola) hat $S$. maximum die größte Frequenz an den Lokalitäten und 
in den geomorphologischen Einheiten. Diese Art ist wahrscheinlich weiter verbreitet und zahlreicher als bislang angenommen wurde (es wurde nur ein Teil des publizierten Materials revidiert). UnterspezifischenUmständen kommt die Art auch in niedrigeren Einheiten vor - wie z. B. im Slowakischen Karst (Zádielska dolina, ab $260 \mathrm{~m}$ ü. M. in einem Canon). Auf der anderen Seite scheint $S$. monticola nicht so weit verbreitet zu sein, wie früher angenommen wurde.

\section{Höhenzonenverbreitung in der Slowakei}

S. variegatum wurde zwischen 120 und $990 \mathrm{~m}$ über dem Meeresniveau gefunden. Die meisten Fundorte befinden sich zwischen 200 und $900 \mathrm{~m}$ (Fig. 6). Das Vorkommen in den Niederungen (Donau, Trnavská tabula, Ipel’ská kotlina) ist selten, bzw. an die Donau mit ihrem besonderen Charakter gebunden. Das Vorkommen von S. variegatum ist signifikant abhängig von der Meereshöhe (Polynom des 2. Grades: $\left.\mathrm{F}_{2,488}=6,42 ; \mathrm{p}=0,002\right)$. Der Scheitel der logistischen Wahrscheinlichkeitskurve befindet sich in $720 \mathrm{~m}$ über Meeresniveau (Fig. 8).

Die Fundorte von $S$. argyreatum befinden sich zwischen 120 und $1490 \mathrm{~m}$ über dem Meeresniveau. Die mei sten Fundorte befinden sich zwischen 200 und $900 \mathrm{~m}$ (Fig. 6).

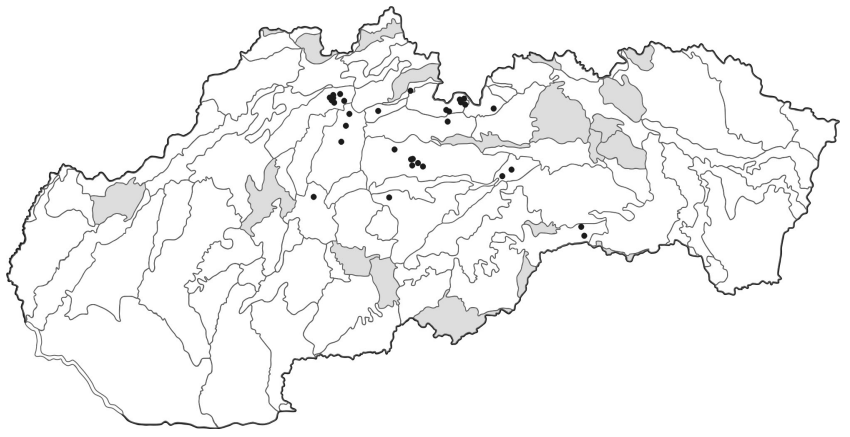

Fig. 4: Die Fundorte von S. maximum in den geomorphologischen Einheiten der Slowakei. Publizierte und eigene Angaben. Hellgrau: Einheiten ohne bekannte Kriebelmückenfundorte.

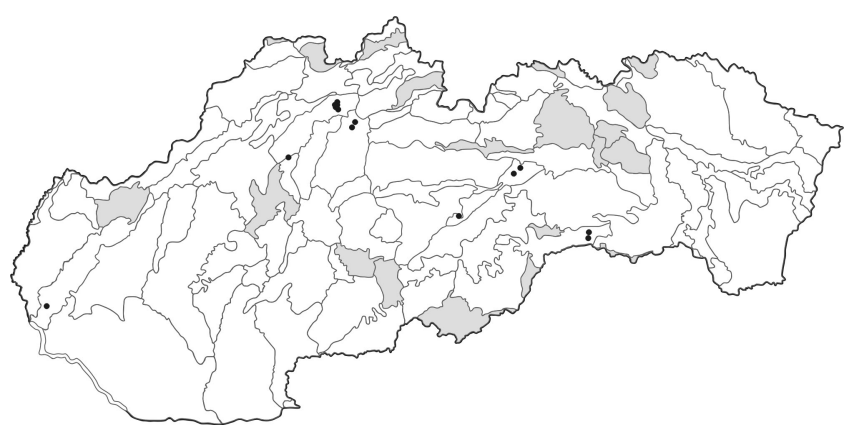

Fig. 5: Die Fundorte von $S$. sp. aff. monticola in den geomorphologischen Einheiten der Slowakei. Publizierte und eigene Angaben. Hellgrau: Einheiten ohne bekannte Kriebelmückenfundorte.

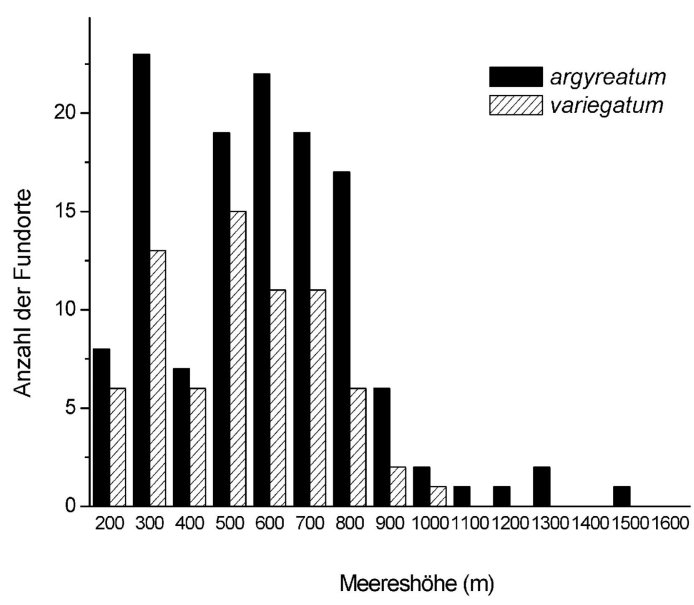

Fig. 6: Anzahl der Fundorte von S. variegatum und S. argyreatum in den Meereshöhenzonen. Die obere Grenze der 100 m breiten Zonen wird angegeben. 


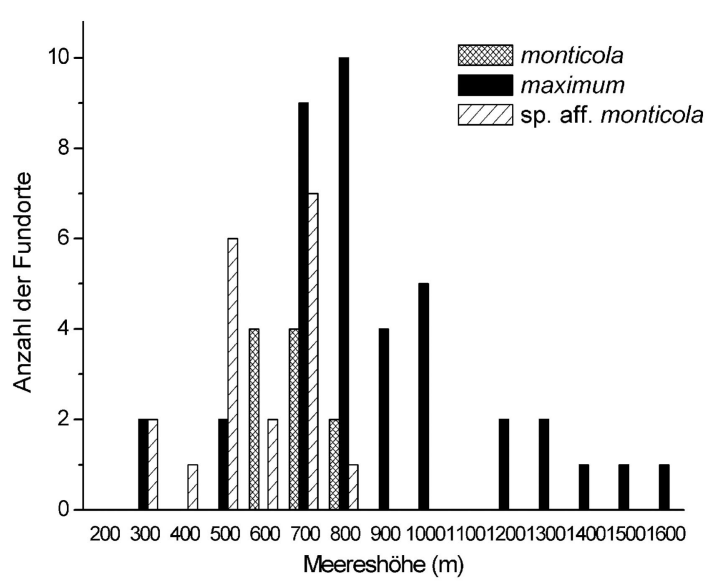

Fig. 7: Anzahl der Fundorte von S. monticola, S. maximum und S. sp. aff. monticola in den Meereshöhenzonen. Die obere Grenze der $100 \mathrm{~m}$ breiten Zonen wird angegeben.
Das Vorkommen in den Niederungen (Borská nížina und Podunajská rovina) ist beschränkt. Die Art lebt in der zentralen Zone von Borská nížina, die bewaldet ist, und ein wellenförmiges Relief mit natürlichen Wasserläufen hat, wo sich auch in höheren Lagen lebende Organismen erhalten konnten, und im Fall von Podunajská rovina handelt es sich um die Donau, die hier noch den Charakter eines Gebirgsflußes hat, was sich auch in den Kriebelmücken gemeinschaften reflektiert (ILLÉŠová u. a., 1994). Bei dem weiterem Fundort in Podunajská rovina handelt es sich um einen Fund adulter Weibchen in der Nähe des Gebirges. Das Vorkommen von S. argyreatum ist signifikant von der Meereshöhe abhängig (Polynom des 2. Grades: $F_{2,488}=17,45$; $\mathrm{p}<0,0001)$. Der Scheitel der logistischen Wahrscheinlichkeitskurve befindet sich in 820 m über Meeresniveau (Fig. 8).

S. monticola wurde in Meereshöhen zwischen 520 und 720 m gefunden. Am häufigsten ist die Art zwischen 600 und 700 m (Fig. 7). Das Vorkommen von S. monticola ist signifikant abhängig von der Meereshöhe (Polynom des 2. Grades: $F_{2,263}=6,23 ; p=0,002$ ). Die Wahrscheinlichkeitskurve des Vorkommens (Fig. 8) hat den Scheitel in der Meereshöhe $880 \mathrm{~m}$.

S. maximum hat die größte Streubreite der Fundorte - sie wurde in Meereshöhen zwischen 260 und 1580 m gefunden. Die meisten Fundorte gibt es zwischen 600 und $700 \mathrm{~m}$ (Fig. 7). Das Vorkommen von S. maximum ist signifikant abhängig von der Meereshöhe (Polynom des 2. Grades: $\left.\mathrm{F}_{2,263}=49,11 ; \mathrm{p}<0,0001\right)$. Die Wahrscheinlichkeitskurve des Vorkommens (Fig. 8) hat den Scheitel in der Meereshöhe von ungefähr $1200 \mathrm{~m}$. Der scheinbare Widerspruch zwischen der Zone mit den meisten Fundorte und dem Vorkommenswahrscheinlichkeitsmaximum ist durch relativ großen Anteil der Art in den relativ wenigen höher gelegenen Lokalitäten verursacht.

S. sp. aff. monticola wurde in Meereshöhen zwischen 260 und 720 m gefunden. Am häufigsten ist die Art zwischen 600 und 700 m (Fig. 7). Das Vorkommen von S. sp. aff. monticola ist signifikant abhängig von der Meereshöhe (Polynom des 2. Grades: $F_{2,263}=5,14$; $\mathrm{p}=0,006$ ). Die Wahrscheinlichkeitskurve des Vorkommens (Fig. 8) hat den Scheitel in der Meereshöhe $810 \mathrm{~m}$.

Die Lage des Scheitels der Wahrscheinlichkeitskurve des Vorkommens ist vor allem bei den Arten mit geringer Anzahl der Fundorte ( $S$. monticola und $S$. sp. aff. monticola) scheinbar nach rechts - außerhalb der wirklichen Fundortstreubreite - verschoben. Beim Vergleich des Scheitels aller fünf Arten zeigt sich, daß S. variegatum niedrigere Lagen und S. maximum höhere Lagen als alle anderen Arten bewohnen. Die Unterschiede zwischen den restlichen drei Arten sind gering und bedürfen weiterer Klärung. Ebenso wären mehr Angaben zur Verbreitung von $S$. monticola und $S$. sp. aff. monticola wünschenswert. 

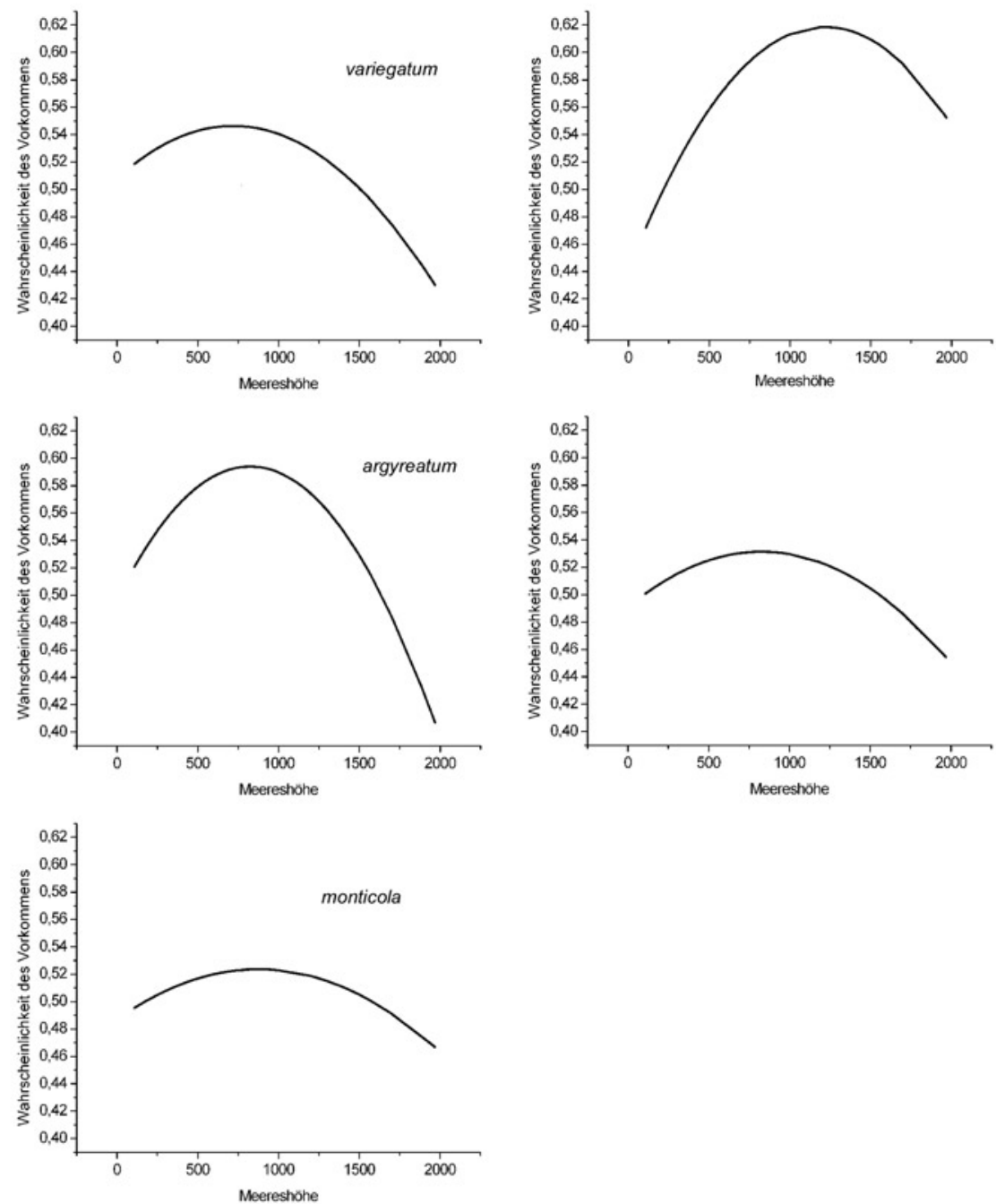

Fig. 8: Wahrscheinlichkeit des Vorkommens von studierten Arten in Abhängigkeit von der Meereshöhe. Logistische Funktion der quadratischen Regression - siehe Material und Methoden.

\section{Danksagung}

Der Autor dankt Herrn Prof. Ladislav Jedlička für die Hilfe mit der Bearbeitung des Manuskripts, sowie für die Bereitstellung der faunistischen Datensammlung der Kriebelmücken in der Slowakei und Herrn Prof. Jozef Halgoš für die Bereitstellung des Materials aus Griechenland. Die Arbeit ist mit der finanziellen Unterstützung des Grants VEGA 1/2349/05 entstanden. 


\section{Literatur}

Bastl, I.; Nagy, S.; MészÁros, J. \& Krnové, M. 1992: Zoobentos, ichtyofauna a helmintofauna rýb tokov NP Malá Fatra a návrh opatrení na ochranu hydrofauny. - Ochrana prírody, 1: 117-167.

BAss, J. 1998: Last-instar larvae and pupae of the Simuliidae of Britain and Ireland: A key with brief ecological notes. - Freshwater Biological Association, The Ferry House: 104 S.

Bernard, M. R. 1970: Simulium (Eusimulium) latinum (Rubzov, 1962) et Simulium (Simulium) maximum (KNOz, 1961) (Diptera, Simuliidae) espèces nouvelles pour la faune de France. - Cashier O.R.S.T.O.M., séries Entomologie médicale et Parasitologie, 8: 319-322.

Bulánková, E. \& Halgoš, J. 1995: Príspevok k poznaniu druhov vybraných čeladí dvojkrídlovcov (Diptera) pohoria Vtáčnik. - Rosalia, 10: 145-150.

BulánKová, E.; Krno, I. \& Halgoš, J. 2000: Makrozoobentos ako indikátor odprírodnenia povodia Hrona v regióne Žiarskej kotliny. - Správy Slovenskej zoologickej spoločnosti 18: 81-94.

Bulánková, E.; Halgoš, J. \& Illéšová, D. 2001: Changes of the Diptera taxoceonoses in the longitudinal profile of the Gidra stream basin (the Little Carpathians). - Acta Universitatis Carolinae-Biologica 45: $19-24$.

BulánKová, E.; Halgoš, J. \& Illéšová, D. 2002: Spoločenstvá dvojkrídlovcov (Diptera, excl. Chironomidae) dvoch tokov Bukovských vrchov (Východné Karpaty). - Acta Facultatis Ecologiae 9: 79-84.

Crosskey, R. W. 1991: On Simulium xanthinum, a curious yellow-bodied blackfly of the southwestern Palearctic region (Diptera: Simuliidae). - Journal of Natural History 25: 691-702.

Crosskey, R. W. \& Crosskey, M. E. 2000: An investigation of the blackfly fauna of Andalusia, southern Spain (Diptera: Simuliidae). - Journal of Natural History 34: 895-951.

Crosskey, R. W. \& Howard, T. M. 2004: A revised taxonomic and geographical inventory of world blackflies (Diptera: Simuliidae). - http://www.nhm.ac.uk/entomology/projects/blackflies/index.html, 13.2.2004: 86 S.

Dinulescu, G. 1966: Fauna Republicii Socialiste Romania. Insecta, Diptera, Fam. Simuliidae. - Editura Academiei Republicii Socialiste Romania, Bucuresti: 604 S.

DušinskÝ, R.; Kúdela, M.; STloukalová \& JedličKa, L. 2006: Use of inter-simple sequence repeat (ISSR) markers for discrimination between and within species of blackflies (Diptera: Simuliidae). - Biologia, Bratislava, 61: 299-304

Dову, J. M. 1963: A propas de Simulium rheophila Knoz, 1961 et de Simulium dorieri Doby \& Rault, 1960. Presence en Autriche de Simulium maximum Knoz, 1961. - Bulletin de la Société Zoologique de France 88: 131-145.

GlathaAR, R. 1978: Verbreitung und Ökologie der Kriebelmücken (Diptera, Simuliidae) in der Schweiz. - Vierteljahresschrift der Naturforschenden Gesellschaft in Zürich 123: 71-124.

Halgoš, J. \& JedličKa, L. 1974: Rozšírenie muškovitých (Diptera, Simuliidae) v Malých Karpatoch. - Acta rerum naturalium Musei Nationalis Slovaciae Bratislava 19: 173-183.

Harvančík, S. \& Topercer, V. 1985: Hematofágne dvojkrídlovce (Diptera) napádajúce vtákov na západnom Slovensku. - In: PAČUtA, M. \& STOlláR, Š. (Hrsg.): Zborník Organizmy a prostredie, Nitra: 149-152.

ILLÉŠovÁ, D. 1989: Age structure of larvae and number of generations in the populations of Odagmia monticola (Friederichs, 1920), O. argyreata (Meigen, 1838) and O. variegata (Meigen, 1818) (Diptera, Simuliidae) in Velká Fatra Mountains. - Biológia, Bratislava, 44: 953-964.

IlléŠovÁ, D. \& Halgoš, J. 1999: The structure of blackflies communities (Diptera, Simuliidae) in the Polana Biospheric Reserve. - Dipterologica bohemoslovaca 9: 71-76.

IlLÉŠová, D. \& HaLGoš, J. 2002: Contribution to knowledge of black flies (Diptera, Simuliidae) in Eastern Slovakia. - Acta Zoologica Universitatis Comenianae 44: 85-90.

Illéšová, D. \& Halgoš, J. 2004: Blackflies (Diptera: Simuliidae) of the Turiec River. - Acta Zoologica Universitatis Comenianae 46: 59-63. 
IllÉŠová, D. \& JedličKa, L. 1992: Black fly communities of the basin of Bystrica in Velká Fatra Mts. (West Carpathians) (Diptera: Simuliidae). - Biológia, Bratislava 47: 627-640.

IlléŠovÁ, D.; JedličKa, L. \& Halgoš, J. 1994: Black flies of the Danube inland delta (Diptera, Simuliidae). - Biologia, Bratislava 49: 223-227.

IlléŠová, D.; Halgoš, J. \& JedličKa, L. 1995. Black flies (Diptera, Simuliidae) in the lower basin of the river Morava. - Biologia, Bratislava 50: 163-169.

IlléšovÁ, D.; Halgoš, J. \& Krno, I. 2000: Blackfly communities (Diptera, Simuliidae) in mountain streams of the High Tatra Mts. - Biologia, Bratislava 55: 177-184.

Jankovskij, A. V. 2002: Opredelitel' moschek (Diptera: Simuliidae) Rossii i sopredel'nych territorij (byvschevo SSSR). - Zoologitscheskij institut Rossijskoj akademii nauk, Sankt-Peterburg: 570 S.

JedLIČKA, L. 1970: Die Verbreitung der Kriebelmücken (Diptera, Simuliidae) in der Südwestslowakei. Acta rerum naturalium Musei Nationalis Slovaciae Bratislava 16: 45-56.

JedučKa, L. 1976: Black flies (Diptera, Simuliidae) spread in Middle Slovakia. - Acta Facultatis Rerum Naturalium Universitatis Comenianae-Zoologia 20: 97-127.

JeDLIČKA, L. 1982: Muškovité rieky Belej a niektorých jej prítokov (Diptera, Simuliidae). - Zborník TANAP 23: 203-209.

JedličKa, L. 1984: Simuliidae. - In: ČEpelák, J. (Hrsg.): Diptera Slovenska I. - Veda, Bratislava: 74-83.

JedlčKa, L.; Stloukalová, V. \& Halgoš, J. 2001: Biodiverzita muškovitých územia Slovenska (Diptera: Simuliidae). - Entomofauna carpathica 13: 25-34.

JedučKa, L. \& Trpiš, M. 1971: Notes on the distribution of black flies (Diptera: Simuliidae) in East Slovakia. - Acta rerum naturalium Musei Nationalis Slovaciae Bratislava 17: 109-120.

Knoz, J. 1961: Př́spěvek k poznání druhu Odagmia monticola (Fried.) 1920 (Diptera, Simuliidae) z ČSSR. - Folia zoologica 10: 101-115.

Knoz, J. \& JedličKa, L. 1997: Simuliidae. - In: Chvála, M. (Hrsg.): Check List of Diptera (Insecta) of the Czech and Slovak Republics. - Karolinum, Praha: 42.

KRNO, I. 1978: Zoobentos rieky Revúcej a jej prítokov. - Biologické práce, 24 (2): 61-124.

KRNO, I. 1982: Štruktúra a dynamika makrozoobentosu riečky Lupčianky a jej prítokov (Nízke Tatry). - Biologické práce, 28 (2): 3-128.

KRNO, I. 1995: Zmeny v štruktúre makrozoobentosu rieky Orava za obdobie 1950-1994. - In: BeLANsKÝ, P. \& Removčí́ková, O. (Hrsg.): Rieka Orava a jej prírodné hodnoty: 29-37.

KRno, I.; Bulánková, E. \& Halgoš, J. 1993: Present condition of water cleanness in the River Váh (LiskováLubochňa) in the vicinity of Ružomberok. - Acta Zoologica Universitatis Comenianae 37: 63-68.

Krno, I.; Bulánkoví, E. \& Halgoš, J. 1994a: Macrozoobenthos of the Morava river basin and tributaries of the Morava. - Ekológia Bratislava 13 (suppl. 1): 63-76.

Krno, I.; Illéšová, D. \& Halgoš, J. 1994b: Temporal fauna of the Gidra Brook (Little Carpathians, Slovakia). - Acta Zoologica Universitatis Comenianae 38: 35-46.

Kuвovčík,V. \& Noviкмec, M. 2001: Preimaginal stages of Diptera from different substrates of the Zbojský potok stream (Poloniny National Park). - Acta Universitatis Carolinae-Biologica 45: 89-96.

Kúdela, M. \& JeDLIČKA, L. 2002: Variability of the respiratory surface area of pupae in Simulium monticola species group (Diptera, Simuliidae). - Limnologica 32: 195-200.

Mazúr, E. \& Lukniš, M. 1978: Regionálne geomorfologické členenie SSR. - Geografický časopis, 30: 101-125.

MinÁŘ, J. \& Christova, T. 1971: Materialy po izutscheniju fauny moschek i komarov v Bolgarii (Diptera: Simuliidae, Culicidae). - Trudy Nautschno-Issledovatel'skovo Instituta Epidemiologii i Mikrobiologii 15: 245-247.

Novák, V. J. 1956: Príspěvek k poznání muchniček (Simuliidae, Diptera) ČSR. - Vestník československé zoologické společnosti, 20: 224-248. 
OвR, S. 1956: Hydrobiologický výzkum povodí Oravy s ohledem na čistotu vody. - Práce Brnenské základny ČSAV, 28: 377-445.

Papp, L. 2001: Simuliidae. - In: PApp, L. (Hrsg.): Checklist of the Diptera of Hungary. Hungarian Natural History Museum, Budapest: 87-89.

Rivosecchi, L. 1978: Simuliidae. Diptera. Nematocera. Fauna d'Italia. Vol. XIII. - Edizioni Calderini, Bologna: $538 \mathrm{~S}$.

Rubzow, I. A. 1956: Moški (sem. Simuliidae). Fauna SSSR, Nasekomyie dvukrylyie, tom VI, vyp. 6, 2-e izdanie. - Izdatel'stvo Akademii nauk SSSR, Moskva, Leningrad: 860 S.

Rubzow, I. A. 1964: 14. Simuliidae (Melusinidae). - In: Lindner, E. (Hrsg.). Die Fliegen der Palaearktischen Region, Band III, 4. - E. Schweizerbart'sche Verlagsbuchhandlung, Stuttgart: 1-670.

Rubzow, I. A. 1967: Über die Kriebelmücken Ungarns. - Annales Historico-Naturales Musei Nationalis Hungarici, Pars Zoologica 59: 303-318.

Rubzow, I. A. 1964: 14. Simuliidae (Melusinidae). Pp. 1-670. - In: Lindner, E. (ed.). Die Fliegen der Palaearktischen Region, Band III, 4. E. Schweizerbart'sche Verlagsbuchhandlung, Stuttgart.

Rubzow, I. A. 1967: Über die Kriebelmücken Ungarns. - Annales Historico-Naturales Musei Nationalis Hungarici, Pars Zoologica 59: 303-318.

SeITZ, G. 1992: Verbreitung und Ökologie der Kriebelmücken (Diptera: Simuliidae) in Niederbayern. - Lauterbornia 11: 1-230.

Tschubareva, L. A. \& Jankovskij, A. V. 1992: Novyj vid moschek Odagmia laplandica Chubareva et Yankovsky sp. n. iz Laplandskogo zapovednika (Kol'skij poluostrov) (Diptera: Simuliidae). - Parazitologija, 26: 326-328.

Thalhammer, J. 1900: Ordo. Diptera. - In: A Magyar Birodalom Állatvilága. A Magyar Birodalomból eddig ismert állatok rendszeres lajstroma. Fauna Regni Hungariae. Animalium Hungariae hucusque cognitorum enumeratio systematica. A K. M. Természettudományi Társulat, Budapest: $76 \mathrm{~S}$.

ZwICK, H. 1974: Faunistisch-ökologische und taxonomische Untersuchungen an Simuliidae (Diptera), unter besonderer Berücksichtigung der Arten des Fulda-Gebietes. - Abhandlungen der Senckenbergischen Naturforschenden Gesellschaft, 533. Verlag Waldemar Kramer, Frankfurt am Main: 116 pp.

ZwICK, H. 1993: Zum Stand der Taxonomie und Determination einheimischer Kriebelmücken (Diptera: Simuliidae). - In: Tıмм, T. \& Rüнм, W. (Hrsg.): Beiträge zur Taxonomie, Faunistik und Ökologie der Kriebelmücken in Mitteleuropa (Diptera, Simuliidae). - Essener Ökologische Schriften, Bd. 2. Westarp Wissenschaften, Essen: $116 \mathrm{~S}$.

\section{Anschrift des Verfassers:}

MATÚŠ KúdelA

Institut für Zoologie

Mlynská dolina

SK-84215 Bratislava, Slowakei

e-mail: kudela@fns.uniba.sk 